\title{
VALOR DE LA RESONANCIA MAGNETICA PRECOZ EN EL DIAGNOSTICO DE FRACTURAS DE ESCAFOIDES
}

\section{Drs. Oscar Contreras $O^{(1)}$, Alvaro Burdiles $O^{(1)}$, Luis Irribarra $T^{(2)}$, Daniel Apablaza $C^{(2)}$.}

1. Servicio de Radiología. Hospital Clínico Pontificia Universidad Católica de Chile.

2. Servicio de Traumatología. Hospital Clínico Pontificia Universidad Católica de Chile.

\begin{abstract}
Introduction: In cases of clinically suspected scaphoid fracture, without radiographic evidence, immobilization and symptomatic treatment is recommended. The use of MRI would define how many of this cases actually present scaphoid fracture, modifying treatment. Materials and methods: In 15 patients with clinically suspected scaphoid fracture and negative radiograph, MRI exams were done 9 days or earlier after trauma. The study included coronal turbo-spin-echo (TSE) T1 weighted images and coronal short tau inversion recovery (STIR) sequences. Results: MRI showed occult scaphoid fractures in 5 patients (33\%), with the combination of T1 and STIR sequences. Patients with negative radiograph and $M R I$ studies reintegrated to normal activities in a period shorter than 2 weeks after trauma. As a consequence there was a reduction in economic costs of at least $50 \%$ considering only radiologic studies, and decreased number of medical consultation. Discussion: MRI early after trauma show a high sensitivity detecting scaphoid fractures radiographically occult, allowing early diagnosis and treatment, avoiding unnecessary immobilization and reducing costs in those patients without fracture.
\end{abstract}

Keywords: Fracture, Scaphoid, Radiograph, MRI.

Resumen. Introducción: La sospecha clínica de fractura de escafoides sin confirmación radiográfica, habitualmente lleva a inmovilización y tratamiento

Contreras 0 . y cols. Valor de la resonancia magnética precoz en el diagnóstico de fracturas de escafoides. Rev Chil Radiol 2004; 10: 12-14.

Correspondencia: Dr. Alvaro Burdiles 0. Marcoleta 367, Santiago. Fono 3543884.

email: aburdiles@mac.com sintomático. La resonancia magnética (RM) precoz permite definir cuales casos presentan realmente fractura, modificando la conducta terapéutica. Material y métodos: En 15 pacientes con sospecha clínica de fractura de escafoides y radiografía normal, se realizó RM dentro de un plazo no mayor a 9 días después del trauma. Se utilizaron secuencias T1 turbo-spinecho (TSE) coronal y short-tau-inversion recovery (STIR) coronal. Resultados: La RM demostró fracturas ocultas de escafoides en 5 pacientes (33\%), todas ellas fueron detectadas con una combinación de secuencias STIR y T1. Los pacientes con radiografía y RM negativos para fractura se reintegraron a sus actividades habituales en un plazo menor a dos semanas. Esto se tradujo en reducción de al menos $50 \%$ de costos en exámenes radiológicos, además de disminuir el número de consultas de control y ausentismo laboral. Conclusión: La RM precoz tiene alta sensibilidad para la detección de fracturas de escafoides radiográficamente ocultas y permite un diagnóstico definitivo y tratamiento oportuno, evitando inmovilización innecesaria en pacientes sin fractura, llevando a una significativa reducción de costos.

Palabras claves: Escafoides, Fractura, Radiografía, Resonancia magnética.

\section{Introducción}

Las fracturas de escafoides no detectadas por el estudio radiológico inicial son frecuentes y pueden dejar importantes secuelas, en el corto y largo plazo, por otra parte existe un porcentaje variable de exámenes falsos positivos.

En los casos de sospecha clínica de fractura de escafoides sin evidencia radiográfica, se realiza en forma habitual inmovilización y tratamiento de los síntomas, con seguimiento clínico y radiográfico.

En el control radiográfico en ocasiones se requieren hasta 6 semanas para que la fractura sea 
visible. Por ello se han planteado otras modalidades de imágenes para la evaluación de estos pacientes.

La resonancia magnética $(\mathrm{RM})$, con alta sensibilidad para detectar fractura, al ser efectuada en forma precoz puede definir los casos que realmente la presentan, modificando por tanto la conducta a seguir, con importantes consecuencias, especialmente en lo referente a costos.

\section{Objetivo}

Evaluar el valor diagnóstico de la RM efectuada precozmente en pacientes con sospecha clínica de fractura de escafoides y radiografía inicial normal.

\section{Materiales y métodos}

Se estudió en forma prospectiva a 15 pacientes que consultaron en el Servicio de Urgencia de nuestra institución, entre Septiembre de 2001 y Mayo de 2003. Todos tenían sospecha clínica de fractura de escafoides y radiografía inicial normal en las 4 proyecciones clásicas. Este grupo incluye 10 hombres y 5 mujeres, con un promedio de edad de 28,4 años (Rango: 12 a 56), 9 con dolor a izquierda y 6 a derecha (Tabla I).

Los criterios de inclusión considerados fueron: Cualquier edad, clínica positiva y trauma reciente, entendiendo como tal el ocurrido dentro de dos semanas. Se consideró evidencia clínica la presencia de tres de los cuatro siguientes signos: compresión axial, dolor en la tabaquera anatómica, edema en la tabaquera anatómica o dolor en el tubérculo (Tabla II).

Los estudios de RM se realizaron dentro de un plazo no mayor a 9 días de ocurrido el trauma.

Se utilizaron secuencias ponderadas en T1 turbo-spin-echo (TSE) (TR 500/TE 17) coronal y short tau inversion recovery (STIR) (TR 1500/TE 15) coronal, con espesor de corte de $3 \mathrm{~mm}$, en un equipo Phillips, modelo Gyroscan Intera T15. El tiempo promedio de estudio fue aproximadamente 10 minutos.
Los estudios fueron evaluados por un radiólogo (O.C.) experto en resonancia magnética.

Si el paciente no presentaba evidencias de fractura en RM, se realizó tratamiento sintomático y control en dos semanas. En los casos con radiografía negativa, pero en los cuales la RM era positiva, se realizó inmovilización con yeso por 6 semanas.

\section{Resultados}

La RM demostró fracturas ocultas de escafoides en 5 pacientes (33\%) (Figura 1) y en uncaso demostró fracturas de radio distal y hueso semilunar, en ausencia de fractura de escafoides (Figura 2). Todas las fracturas se detectaron con una combinación de secuencias STIR y T1.

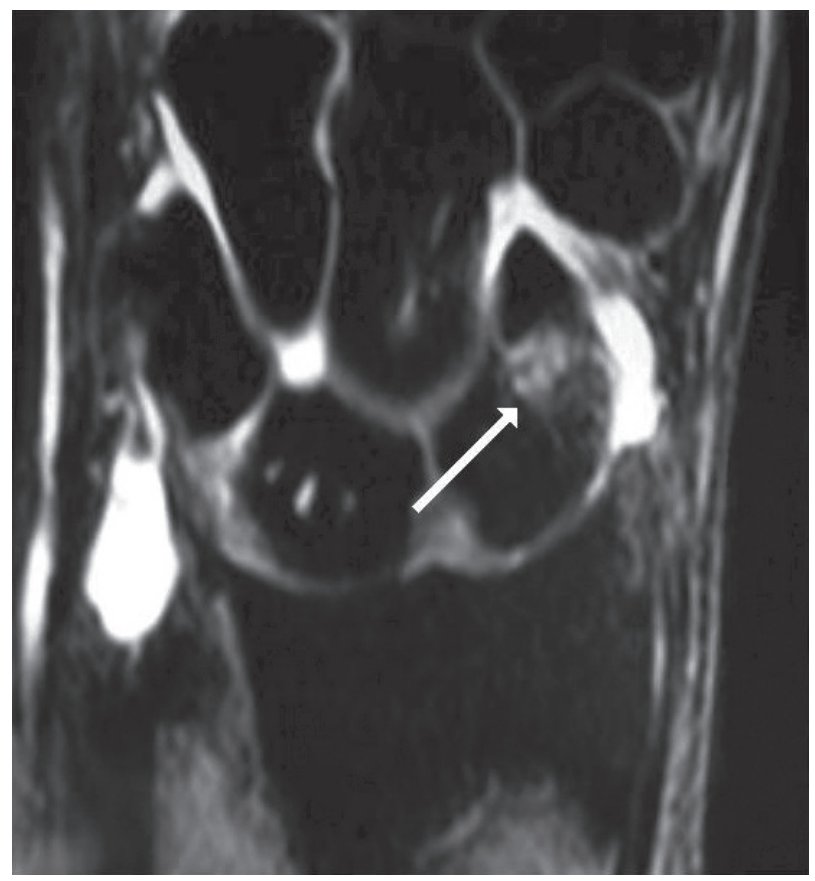

Figura 1. RM ponderada en secuencia STIR, coronal. Se observa imagen de hiperseñal en la cintura del escafoides (flecha). En T1 fue visible como imagen lineal compatible con fractura.

Tabla I. Distribución de pacientes y fracturas por sexo, edad y lado.

\begin{tabular}{lrlll} 
& \multicolumn{1}{c}{ Sexo } & Edad (años) & Lado \\
Pacientes & 10 & Masc. 5 Fem. & Promedio: 28,4 Rango: 12-56 & 9 Izq. 6 Der. \\
Fracturas & 5 Masc. & Promedio: 28,2 Rango: 12-52 & 3 Izq. 2 Der.
\end{tabular}

Tabla II. Sensibilidad de síntomas y signos clínicos.

Síntomas y signos Compresión axial Dolor tabaquera Edema tabaquera Dolor tubérculo

$\begin{array}{lllll}\text { Sensibilidad } & 60 \% & 80 \% & 60 \% & 60 \%\end{array}$




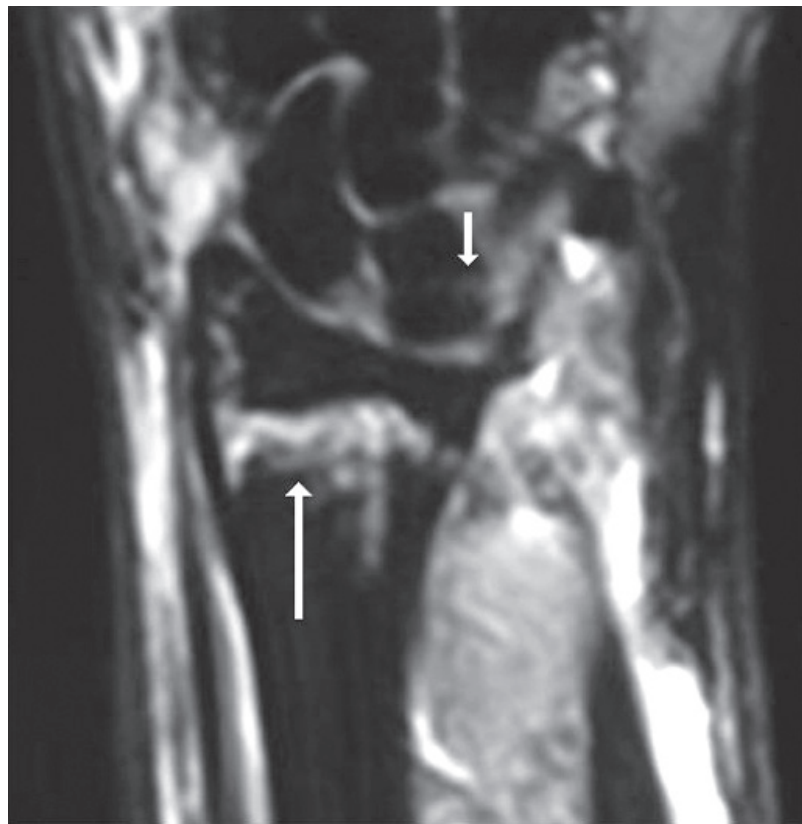

Figura 2. RM ponderada en secuencia STIR, coronal. Se observa una imagen de hiperseñal lineal de disposición transversa en semilunar (flecha corta) y otra en banda transversal en radio distal (flecha larga). Estas fracturas no fueron visibles en radiografías del mismo día.

\section{Discusión}

Diversas publicaciones han hecho notar la alta sensibilidad de la RM para la detección de fracturas en escafoides no detectadas por el examen radiológico inicial ${ }^{(1-8)}$, sin embargo, nos parece de gran importancia considerar el aspecto económico en el manejo de estos pacientes, que también ha sido discutido en la literatura ${ }^{(3,7)}$

Compararemos los costos del seguimiento clínico y radiológico habitual, con el protocolo de estudio con RM. Para ejemplificar mostraremos precios de referencia en uno de los centros de nuestra institución.

El paciente consulta en el Servicio de Urgencia (\$25.000 en horario hábil, \$37.500 en horario no hábil). Si existe sospecha clínica se realizará estudio con radiografía de escafoides (4 proyecciones) (\$24.000 en horario hábil, y \$ 36.000 en horario no hábil). Si la radiografía es negativa, pero existe sospecha clínica se colocará un yeso de inmovilización (\$33.000 en horario hábil, \$49.501 en horario no hábil).

En dos semanas se realiza el primer control, con retiro de yeso $(\$ 19.800)$ y una nueva serie de radiografías (\$24.000). Si la radiografía aún es negativa y si persiste la sospecha clínica, se coloca nuevamente un yeso (\$24.436) y se realizaran nuevos controles a la cuarta y sexta semana. El costo total de este estudio y seguimiento varía entre $\$ 262.272$ (horario hábil de primera consulta) y \$303.273 (horario no hábil) (Tabla III).
Nuestra modificación de protocolo incluye, luego de la consulta inicial en el Servicio de Urgencia, si existe sospecha clínica de fractura de escafoides con estudio radiográfico negativo, la utilización de $\mathrm{RM}$ con protocolo especial (\$70.000). Si este examen es negativo se utiliza en ocasiones un inmovilizador de pulgar (\$7.000). Posteriormente se realiza un control a las 2 semanas $(\$ 19.800)$. El costo total de este estudio y seguimiento varía entre $\$ 145.800$ (horario hábil de primera consulta) y $\$ 170.300$ (horario no hábil) (Tabla IV).

\section{Tabla III. Costos de sospecha clínica de fractura no} confirmada en estudio radiológico. (*)

Consulta

$\begin{array}{lll}\text { Servicio de Urgencia } & \$ 25.000 \text { a } & \$ 37.500 \\ \text { Radiografía } & \$ 24.000 \text { a } & \$ 36.000\end{array}$

Colocación de yeso $\quad \$ 33.000$ a $\$ 49.501$

Control (2a semana) y

retiro de yeso $\$ 19.800$

Radiografía $\$ 24.000$

Colocación de yeso \$24.436

Control (4a semana) y

retiro de yeso $\$ 19.800$

Radiografía $\$ 24.000$

Colocación de yeso \$24.436

Control (6a semana) y

retiro de yeso $\$ 19.800$

Radiografía $\$ 24.000$

TOTAL

$\$ 262.272$ a $\$ 303.273$

$\left.{ }^{*}\right)$ Valores de referencia Fonasa nivel 3. Variación de primera consulta corresponde a horario de atención.

Tabla IV. Costos de sospecha clínica estudiada con RM inicial.

Consulta

Servicio de Urgencia $\$ 25.000$ a $\$ 37.500$

Radiografía

Resonancia magnética

Inmovilizador pulgar

Control (2a semana)

$\$ 24.000$ a $\$ 36.000$

$\$ 70.000$

$\$ 7.000$

$\$ 19.800$

TOTAL

$\$ 145.800$ a $\$ 170.300$

$\left(^{*}\right)$ Valores de referencia Fonasa nivel 3. Variación de primera consulta corresponde a horario de atención. 
Esto implica que utilizando nuestro protocolo, se puede obtener un diagnóstico definitivo precoz y un tratamiento oportuno, con una reducción del costo total de aproximadamente $44 \%$. Además logramos evitar la innecesaria inmovilización de pacientes que no presentan fractura, con sus eventuales complicaciones.

Se debe considerar además que los pacientes con diagnóstico definitivo precoz negativo para fractura pueden reintegrarse tempranamente a sus actividades, disminuyendo el ausentismo laboral asociado. Estos pacientes se reintegraron a sus actividades habituales en un plazo promedio de 14 días (intervalo entre 10 y 21 días), sin presentar sintomatología, y sin requerir uso de medicamentos analgésicos. Además en ellos no fue necesario realizar estudios de imagenología diagnóstica adicionales.

Se ha hecho mención en algunas publicaciones respecto de la utilidad de la ultrasonografía de alta resolución ${ }^{(10)}$ en la evaluación de fracturas de escafoides. Aun cuando esta técnica es de mayor disponibilidad y de menor costo, sus resultados no ha sido validados en el diagrama de flujo del manejo de pacientes con sospecha clínica de fractura de escafoides.

\section{Conclusión}

La RM tiene una alta sensibilidad para la detección de fracturas de escafoides radiográficamente ocultas. Ello permite un diagnóstico definitivo temprano y un tratamiento oportuno, evitando inmovilización innecesaria en pacientes que no presentan fractura, llevando a una significativa reducción en los costos.

En nuestra opinión ante la sospecha clínica de fractura de escafoides y radiografía normal la evaluación inicial debe ser seguida por RM inmediata.

\section{Bibliografía}

1. Raby N. Magnetic resonance imaging of suspected scaphoid fractures using a low field dedicated extremity MR system. Clin Radiol 2001; 56:316-320.

2. Hunter JC, Escobedo EM, Wilson AJ, et al. MR imaging of clinically suspected scaphoid fractures. Am J Roentgenol 1997; 168:1287-1293.

3. Kukla C, Gaebler C, Breitenseher MJ et al. Occult fractures of the scaphoid. The diagnostic usefulness and indirect economic repercussions of radiography versus magnetic resonance scanning. J Hand Surg [Br] 1997; 22:810-813.

4. Gaebler C, Kukla C, Breitenseher M, et al. Magnetic Resonance Imaging of occult Scaphoid Fractures. The Journal of Trauma: Injury, Infection and critical care 1996; 41:73-76.

5. Perron A, Brady W, Keats T, Hersh R. Orthopedic Pitfalls in the ED: Scaphoid Fracture. Am J Emerg Med 2001; 19:310-316.

6. Bretlau T, Christensen OM, Edstrom P, et al. Diagnosis of scaphoid fracture and dedicated extremity MRI. Acta Orthop Scand 1999; 70: 504-508.

7. Dorsay T, Major N, Helms C. Cost-Effectiveness of immediate MR Imaging versus traditional follow-up for revealing radiographically occult scaphoid fractures. AJR 2001; 177:1257-1263.

8. Fowler C, Sullivan B, Williams LA, et al. A comparison of bone scintigraphy and MRI in the early diagnosis of the occult scaphoid waist fracture. Skeletal Radiol 1998; 27:683-687.

9. Saxena P, McDOnald R, Gull S, Hyder N. Diagnostic scanning for suspected scaphoid fractures: an economic evaluation based on costminimisation models injury. Int J Care Injured 2003; 34: 503-511.

10. Herneth A, Siegmeth A, Bader T. Scaphoid fractures: evaluation with high-spatial-resolution US. Initial results. Radiology 2001; 220:231-235. 